CERN-TH/2000-184

hep-ph/0007142

\title{
The soft-gluon current at one-loop order 尚
}

\author{
Stefano Catani i \\ Theory Division, CERN, CH-1211 Geneva 23, Switzerland \\ and \\ Massimiliano Grazzini \\ Institute for Theoretical Physics, ETH-Hönggerberg, CH-8093 Zurich, Switzerland
}

\begin{abstract}
We study the soft limit of one-loop QCD amplitudes and we derive the processindependent factorization formula that controls the singular behaviour in this limit. This is obtained from the customary eikonal factorization formula valid at tree (classical) level by introducing a generalized soft-gluon current that embodies the quantum corrections. We compute the explicit expression of the soft-gluon current at one-loop order. It contains purely non-abelian correlations between the colour charges of each pair of hard-momentum partons in the matrix element. This leads to colour correlations between (two and) three hard partons in the matrix element squared. Exploiting colour conservation, we recover QED-like factorization for the square of the matrix elements with two and three hard partons.
\end{abstract}

CERN-TH/2000-184

June 2000

\footnotetext{
*This work was supported in part by the EU Fourth Framework Programme "Training and Mobility of Researchers", Network "Quantum Chromodynamics and the Deep Structure of Elementary Particles", contract FMRX-CT98-0194 (DG 12 - MIHT).

$\dagger$ On leave of absence from INFN, Sezione di Firenze, Florence, Italy.

${ }_{\ddagger}^{\ddagger}$ Work supported by the Swiss National Foundation.
} 


\section{Introduction}

Higher-order computations in perturbative QCD can be performed by using three main tools: exact calculations at a fixed order in the QCD coupling $\alpha_{\mathrm{S}}=g_{\mathrm{S}}^{2} / 4 \pi$, analytic resummed calculations and parton shower event generators. At present, the accuracy of these tools is respectively limited to the next-to-leading order (NLO), to next-to-leading logarithmic (NLL) accuracy and to the dominant soft and collinear enhanced contributions. An extensive and updated list of references can be found in Refs. [1, 2].

Apart from important conceptual and technical differences, these tools are based on a common ingredient: the universal factorization properties of QCD amplitudes in the infrared (soft and collinear) region. The lowest-order version of the soft and collinear factorization formulae [3, 4] was indeed exploited [5] to develop these tools to their present theoretical accuracy. Higher-order versions of the factorization formulae are required to progress towards next-to-next-to-leading order (NNLO) calculations, resummation of nextto-next-to-leading logarithmic (NNLL) terms and inclusion of subdominant contributions in parton showers.

In recent years several groups have contributed to extending infrared factorization to higher perturbative orders 6 16].

The general factorization properties of tree-level and loop amplitudes in the limit where two or more partons become collinear were studied in Refs. [6 9]. At tree level, the singular factors for the collinear splitting of one parton into three were explicitly computed in Refs. [6, 7, 10]. The splitting function corresponding to the clustering of four collinear gluons is also known [11]. The one-loop kernels for the collinear splitting of one parton into two were obtained in Refs. 12 14.

The mixed soft-collinear limit [6, 7] can be studied by exploiting the coherence properties of QCD radiation [四]. Using QCD coherence, the singular behaviour in the softcollinear limit can be treated [7] by combining the singular factors that separately control the collinear and soft limits.

The soft limit is physically more involved than the collinear limit. Long-wavelength (soft) gluons can spread the colour flow over large distances, thus leading to (non-local) colour correlations. Kinematics and colour factors turn out to be deeply entangled in the soft-factorization formulae.

The tree-level factorization formulae for the emission of two soft gluons were independently derived in Refs. [15] and [7]. The soft (and collinear) singular behaviour of two-loop amplitudes was studied in Ref. [16].

In this paper we consider the limit in which a soft gluon is radiated from one-loop amplitudes. The limit was first investigated in Refs. [8, 12, 14]. The formalism used by this group is based on the decomposition of the one-loop matrix elements in colour subamplitudes [17]. The colour-subamplitude decomposition depends on the type of external partons, and these authors derived the explicit expressions of the one-loop soft-gluon contribution to colour subamplitudes with $m$ external gluons [12] and with $m$ external gluons plus a $q \bar{q}$ pair 14 . 
In the present paper the soft limit is studied by means of a completely independent and general method. We apply the eikonal approximation and soft-gluon insertion rules to perform infrared factorization directly in colour space. Within this formalism, the soft limit of tree-level amplitudes is described by a factorization formula written in terms of a soft-gluon (or eikonal) current (see Ref. [4] and Sect. 2) that describes colour radiation in the classical approximation. We show that the factorization formula can be extended to loop amplitudes by introducing a generalized soft-gluon current that embodies (nonabelian) quantum corrections. As at tree level, the soft-gluon current only depends on the colour charges and momenta of the external partons in the loop amplitude. We compute the explicit expression of the soft-gluon current at one-loop order.

Our results for the soft limit of one-loop amplitudes agree with those in Refs. [12, 14] for the particular cases considered therein. The one-loop gluon current that we obtain is derived and presented in a general and process-independent way; it can be applied to any one-loop amplitude. In particular, we can easily show that colour and kinematic factors can be completely disentangled in the computation of the soft limit of the square of one-loop matrix elements with two or three external QCD partons. This simplified factorization structure is particularly useful for the NNLO calculation of 2-jet and 3-jet cross sections in $e^{+} e^{-}$annihilation.

Infrared-factorization properties at the lowest perturbative order have been known for a long time [4. Combining the results obtained here with those in Refs. [6 16], the general structure of the infrared singularities at the next perturbative order is also completely and explicitly known. It can be used to improve the accuracy of perturbative QCD calculations.

The paper is organized as follows. In Sect. 2 we recall the known results for the soft limit of tree-level amplitudes. In Sect. 3 we present the process-independent factorization formula at higher perturbative orders and we discuss in detail its features at one-loop order. In Sect. 4 we prove the factorization formula at one-loop order and we derive the explicit expression of the one-loop soft current. In Sect. 5 we apply our results to the squared amplitudes of processes with two or three hard partons.

\section{Soft-gluon factorization at tree level}

We consider a generic scattering process that involves $m$ external QCD partons (massless quarks and gluons) with momenta $p_{1}, \ldots, p_{m}$ and an arbitrary number and type of particles with no colour (photons, leptons, vector bosons, ...). Note that, by definition, we always consider incoming and outgoing parton momenta in the physical region, i.e. any $p_{i}$ is massless, with positive-definite energy (in particular, $p_{i} \cdot p_{j}>0$ ). The corresponding matrix element is denoted by $\mathcal{M}\left(p_{1}, \ldots, p_{m}\right)$ and the dependence on the momenta and quantum numbers of non-QCD particles is always understood.

The matrix element has the following loop expansion:

$$
\mathcal{M}\left(p_{1}, \ldots, p_{m}\right)=\mathcal{M}^{(0)}\left(p_{1}, \ldots, p_{m}\right)+\mathcal{M}^{(1)}\left(p_{1}, \ldots, p_{m}\right)+\ldots
$$

where $\mathcal{M}^{(0)}$ denotes the tree-level contribution, $\mathcal{M}^{(1)}$ denotes the one-loop contribution, and 
the dots stand for higher-loop corrections. Note that we always consider unrenormalized matrix elements. Thus Eq. (1) is a power series expansion in the bare QCD coupling $g_{\mathrm{S}}$ and, in particular, $\mathcal{M}^{(1)}$ is the unrenormalized one-loop amplitude.

We simultaneously regularize ultraviolet and infrared singularities by using dimensional regularization. Apart from introducing the dimensional-regularization scale $\mu$ through the replacement $g_{\mathrm{S}} \rightarrow g_{\mathrm{S}} \mu^{\epsilon}$, the key ingredient of dimensional regularization is the analytic continuation of loop momenta to $d=4-2 \epsilon$ space-time dimensions. Having done this, we are left with some freedom regarding the dimensionality of the momenta of the external particles as well as the number of polarizations of both external and internal particles. This leads to different regularization schemes [18 21] within the dimensional-regularization prescription. The regularization-scheme dependence of one-loop amplitudes was studied in detail in Refs. [22, 23]. All the results on the soft behaviour presented in this paper do not explicitly depend on the dimensional-regularization scheme (see Sect. 1 for a brief discussion of different regularization schemes): the scheme dependence is implicitly embodied in the expressions of the tree-level and one-loop matrix elements $\mathcal{M}^{(0)}$ and $\mathcal{M}^{(1)}$.

The emission of a soft gluon does not affect the momenta and spins of the radiating hard partons. However, it does affect their colour because the gluon always carries away some colour charge, no matter how soft it is. Unlike the case of soft-photon emission in QED, soft-gluon emission thus does not factorize exactly and leads to colour correlations.

To take into account the colour structure without referring to any particular choice of basis colour vectors (such as, for instance, the decomposition in colour subamplitudes [14]), we use a general notation (see e.g. Ref. [24]). The dependence of the matrix element on the colour indices $c_{1}, \ldots, c_{m}$ of the QCD partons is written as

$$
\mathcal{M}_{c_{1}, \ldots, c_{m}}\left(p_{1}, \ldots, p_{m}\right) \equiv\left\langle c_{1}, \ldots, c_{m} \mid \mathcal{M}\left(p_{1}, \ldots, p_{m}\right)\right\rangle
$$

Thus $\left\{\left|c_{1}, \ldots, c_{m}\right\rangle\right\}$ is an abstract basis in colour space and the ket $\left|\mathcal{M}\left(p_{1}, \ldots, p_{m}\right)\right\rangle$ is a vector in this space. According to this notation, the matrix element squared $|\mathcal{M}|^{2}$ (summed over the colours and spins of the partons) can be written as

$$
\left|\mathcal{M}\left(p_{1}, \ldots, p_{m}\right)\right|^{2}=\left\langle\mathcal{M}\left(p_{1}, \ldots, p_{m}\right) \mid \mathcal{M}\left(p_{1}, \ldots, p_{m}\right)\right\rangle
$$

To describe the colour correlations produced by soft-gluon emission, we associate a colour charge $\boldsymbol{T}_{i}$ with the emission of a gluon from each parton $i$. If the emitted gluon has colour index $a\left(a=1, \ldots, N_{c}^{2}-1\right)$, the colour-charge operator is:

$$
\boldsymbol{T}_{i} \equiv\langle a| T_{i}^{a}
$$

and its action onto the colour space is defined by

$$
\left\langle a, c_{1}, \ldots, c_{i}, \ldots, c_{m}\left|\boldsymbol{T}_{i}\right| b_{1}, \ldots, b_{i}, \ldots, b_{m}\right\rangle=\delta_{c_{1} b_{1}} \ldots T_{c_{i} b_{i}}^{a} \ldots \delta_{c_{m} b_{m}}
$$

where $T_{c b}^{a} \equiv i f_{c a b}$ (colour-charge matrix in the adjoint representation) if the emitting parton $i$ is a gluon and $T_{\alpha \beta}^{a} \equiv t_{\alpha \beta}^{a}$ (colour-charge matrix in the fundamental representation with $\left.\alpha, \beta=1, \ldots, N_{c}\right)$ if the emitting particle $i$ is a final-state quark or an initial-state antiquark $\left(T_{\alpha \beta}^{a} \equiv \bar{t}_{\alpha \beta}^{a}=-t_{\beta \alpha}^{a}\right.$, in the case of a final-state antiquark or an initial-state quark). 
The colour-charge algebra is;

$$
T_{i}^{a} T_{j}^{a} \equiv \boldsymbol{T}_{i} \cdot \boldsymbol{T}_{j}=\boldsymbol{T}_{j} \cdot \boldsymbol{T}_{i} \quad \text { if } i \neq j ; \quad \boldsymbol{T}_{i}^{2}=C_{i},
$$

where $C_{i}$ is the Casimir operator, i.e. $C_{i}=C_{A}=N_{c}$ if $i$ is a gluon and $C_{i}=C_{F}=$ $\left(N_{c}^{2}-1\right) / 2 N_{c}$ if $i$ is a quark or antiquark.

Note that, by definition, each vector $\left|\mathcal{M}\left(p_{1}, \ldots, p_{m}\right)\right\rangle$ is a colour-singlet state. Therefore colour conservation is simply

$$
\sum_{i=1}^{m} \boldsymbol{T}_{i}\left|\mathcal{M}\left(p_{1}, \ldots, p_{m}\right)\right\rangle=0 .
$$

We can now recall the behaviour of the tree-level matrix element $\mathcal{M}^{(0)}\left(q, p_{1}, \ldots, p_{m}\right)$ in the limit where the momentum $q$ of the gluon becomes soft. Denoting by $a$ and $\varepsilon^{\mu}(q)$ the colour and the polarization vector of the soft gluon, the matrix element fulfils the following factorization formula [1]

$$
\left\langle a \mid \mathcal{M}^{(0)}\left(q, p_{1}, \ldots, p_{m}\right)\right\rangle \simeq g_{\mathrm{S}} \mu^{\epsilon} \varepsilon^{\mu}(q) J_{\mu}^{a(0)}(q)\left|\mathcal{M}^{(0)}\left(p_{1}, \ldots, p_{m}\right)\right\rangle,
$$

where $\left|\mathcal{M}^{(0)}\left(p_{1}, \ldots, p_{m}\right)\right\rangle$ is obtained from the original matrix element by simply removing the soft gluon $q$. The factor $\boldsymbol{J}_{\mu}^{(0)}(q)$ is the tree-level soft-gluon current

$$
\boldsymbol{J}^{\mu(0)}(q)=\sum_{i=1}^{m} \boldsymbol{T}_{i} \frac{p_{i}^{\mu}}{p_{i} \cdot q},
$$

which depends on the momenta and colour charges of the hard partons in the matrix element on the right-hand side of Eq. (8). The symbol ' $\simeq$ ' means that on the right-hand side we have neglected contributions that are less singular than $1 / q$ in the soft limit $q \rightarrow 0$. Note that Eq. (8) is valid in any number $d=4-2 \epsilon$ of space-time dimensions, and the sole dependence on $d$ is in the overall factor $\mu^{\epsilon}$.

The factorization formula (8) can be derived in a simple way by working in a physical gauge and using the following soft-gluon insertion rules. The coupling of the gluon to any internal (i.e. highly off-shell) parton in the amplitude $\mathcal{M}^{(0)}\left(q, p_{1}, \ldots, p_{m}\right)$ is not singular in the soft limit; it can thus be neglected. The soft-gluon coupling to any external or, in general, nearly on-shell parton with colour charge $\boldsymbol{T}$ and momentum $p$ can be factorized by using the eikonal approximation, that is by extracting the contribution $g_{S} \mu^{\epsilon} 2 p^{\mu} \boldsymbol{T}$ for the vertex and the contribution $1 /(p+q)^{2} \simeq 1 /\left(p^{2}+2 p \cdot q\right)$ for the propagator. Note that the eikonal vertex only depends on the momentum and colour charge of the radiating parton: it does not depend on either the soft momentum or the spin of the parton. Using the eikonal propagator simply amounts to neglecting the terms that are quadratic in the soft momentum.

An important property of the soft-gluon current is current conservation. Multiplying Eq. (9) by $q^{\mu}$, we obtain

$$
q^{\mu} \boldsymbol{J}_{\mu}^{(0)}(q)=\sum_{i=1}^{m} \boldsymbol{T}_{i},
$$

$\S$ More details on the colour algebra and useful colour-matrix relations can be found in Appendix A of Ref. [24]. 
and thus

$$
q^{\mu} \boldsymbol{J}_{\mu}^{(0)}(q)\left|\mathcal{M}^{(0)}\left(p_{1}, \ldots, p_{m}\right)\right\rangle=\sum_{i=1}^{m} \boldsymbol{T}_{i}\left|\mathcal{M}^{(0)}\left(p_{1}, \ldots, p_{m}\right)\right\rangle=0
$$

where the last equality follows from colour conservation as in Eq. (7). Although the factorization formula (8) is most easily derived by working in a physical gauge, the conservation of the soft-gluon current implies that Eq. (8) is actually gauge invariant. Any gauge transformation is equivalent to an addition of a longitudinal component to the polarization vector of the soft gluon through the replacement $\varepsilon^{\mu}(q) \rightarrow \varepsilon^{\mu}(q)+\lambda q^{\mu}$. Nonetheless the factorization formula (8) is invariant under this replacement, because of Eq. (11).

Squaring Eq. (8) and summing over the gluon polarizations leads to the well-known soft-gluon factorization formula at $\mathcal{O}\left(g_{\mathrm{S}}^{2}\right)$ for the squared amplitude [4]:

$$
\left|\mathcal{M}^{(0)}\left(q, p_{1}, \ldots, p_{m}\right)\right|^{2} \simeq-g_{\mathrm{S}}^{2} \mu^{2 \epsilon} 2 \sum_{i, j=1}^{m} \mathcal{S}_{i j}(q)\left|\mathcal{M}_{(i, j)}^{(0)}\left(p_{1}, \ldots, p_{m}\right)\right|^{2},
$$

where the eikonal function $\mathcal{S}_{i j}(q)$ can be written in terms of two-particle sub-energies $s_{i j}=$ $\left(p_{i}+p_{j}\right)^{2}$ as follows

$$
\mathcal{S}_{i j}(q)=\frac{p_{i} \cdot p_{j}}{2\left(p_{i} \cdot q\right)\left(p_{j} \cdot q\right)}=\frac{s_{i j}}{s_{i q} s_{j q}} .
$$

The colour correlations produced at tree level by the emission of a soft gluon are taken into account by the square of the colour-correlated tree-amplitude $\left|\mathcal{M}_{(i, j)}^{(0)}\right|^{2}$ on the right-hand side. This is given by

$$
\begin{aligned}
\left|\mathcal{M}_{(i, j)}^{(0)}\left(p_{1}, \ldots, p_{m}\right)\right|^{2} & \equiv\left\langle\mathcal{M}^{(0)}\left(p_{1}, \ldots, p_{m}\right)\left|\boldsymbol{T}_{i} \cdot \boldsymbol{T}_{j}\right| \mathcal{M}^{(0)}\left(p_{1}, \ldots, p_{m}\right)\right\rangle \\
& =\left[\mathcal{M}_{c_{1} . . b_{i} \ldots b_{j} \ldots c_{m}}^{(0)}\left(p_{1}, \ldots, p_{m}\right)\right]^{*} T_{b_{i} d_{i}}^{a} T_{b_{j} d_{j}}^{a} \mathcal{M}_{c_{1} . . d_{i} \ldots d_{j} \ldots c_{m}}^{(0)}\left(p_{1}, \ldots, p_{m}\right) .
\end{aligned}
$$

\section{Soft-gluon factorization at one loop}

The factorized structure of the soft limit of the QCD amplitudes at tree level can be generalized to higher loops. The soft limit of the one-loop amplitudes is studied in detail in Sect. 6 . In this section we anticipate and discuss the final results.

Our analysis is consistent with the following factorization formula

$$
\left\langle a \mid \mathcal{M}\left(q, p_{1}, \ldots, p_{m}\right)\right\rangle \simeq \varepsilon^{\mu}(q) J_{\mu}^{a}(q, \epsilon)\left|\mathcal{M}\left(p_{1}, \ldots, p_{m}\right)\right\rangle\left[1+\mathcal{O}\left(g_{\mathrm{S}}^{4}\right)\right],
$$

where the symbol ' $\simeq$ ' has the same meaning as in Eq. (8). The matrix element on the right-hand side is the all-loop amplitude in Eq. (II) and the singular dependence on $q$ is embodied in the (unrenormalized) soft-gluon current $J_{\mu}^{a}(q, \epsilon)$, which can be expanded in loop contributions, i.e. in powers of $g_{\mathrm{S}}^{2}$ :

$$
J_{\mu}^{a}(q, \epsilon)=g_{\mathrm{S}} \mu^{\epsilon}\left[J_{\mu}^{a(0)}(q)+g_{\mathrm{S}}^{2} \mu^{2 \epsilon} J_{\mu}^{a(1)}(q, \epsilon)+\ldots\right] .
$$


The term $J_{\mu}^{a(0)}(q)$ is the tree-level current in Eq. (9), the term $J_{\mu}^{a(1)}(q, \epsilon)$ is its one-loop correction, and so forth.

The soft current contains the entire singular dependence in the soft limit and no further approximation is performed in Eq. (15). By this we mean that $J_{\mu}^{a}(q, \epsilon)$ behaves as $1 / q$ (such as at tree level) modulo any possible enhancement proportional to powers of $\ln q$ coming from the loop contributions. In particular, the $\epsilon$-dependence of the current can be evaluated exactly without performing any $\epsilon$-expansion and, thus, by keeping all the powers of $\ln q$ coming from higher-loop contributions of the type $(q)^{\epsilon}=1+\epsilon \ln q+\cdots$.

The discussion in Sect. 4 suggests that the factorization formula (15) is valid to any loop order. Nonetheless, we have included the term $\mathcal{O}\left(g_{\mathrm{S}}^{4}\right)$ on the right-hand side of Eq. (15) to indicate that our explicit proof and calculation do not extend beyond the one-loop order.

Expanding both sides of Eq. (15) to one-loop accuracy and using Eq. (8), we can obtain the factorization formula for the soft limit of the one-loop amplitudes:

$$
\begin{aligned}
\left\langle a \mid \mathcal{M}^{(1)}\left(q, p_{1}, \ldots, p_{m}\right)\right\rangle \simeq g_{S} \mu^{\epsilon} \varepsilon^{\mu}(q)[ & J_{\mu}^{a(0)}(q)\left|\mathcal{M}^{(1)}\left(p_{1}, \ldots, p_{n}\right)\right\rangle \\
& \left.+g_{\mathrm{S}}^{2} \mu^{2 \epsilon} J_{\mu}^{a(1)}(q, \epsilon)\left|\mathcal{M}^{(0)}\left(p_{1}, \ldots, p_{n}\right)\right\rangle\right] .
\end{aligned}
$$

The explicit expression for the one-loop current is

$$
\begin{aligned}
J_{a}^{\mu(1)}(q, \epsilon) & =-\frac{1}{16 \pi^{2}} \frac{1}{\epsilon^{2}} \frac{\Gamma^{3}(1-\epsilon) \Gamma^{2}(1+\epsilon)}{\Gamma(1-2 \epsilon)} \\
& \cdot \quad i f_{a b c} \sum_{i \neq j} T_{i}^{b} T_{j}^{c}\left(\frac{p_{i}^{\mu}}{p_{i} \cdot q}-\frac{p_{j}^{\mu}}{p_{j} \cdot q}\right)\left[\frac{4 \pi p_{i} \cdot p_{j} e^{-i \lambda_{i j} \pi}}{2\left(p_{i} \cdot q\right)\left(p_{j} \cdot q\right) e^{-i \lambda_{i q} \pi} e^{-i \lambda_{j q} \pi}}\right]^{\epsilon} .
\end{aligned}
$$

We remind the reader that in our notation all the incoming and outgoing momenta are in the physical region (any $p_{i}$ has positive-definite energy and $p_{i} \cdot p_{j}>0$ ). Thus the complex factors $e^{-i \pi \lambda_{A B}}\left(\lambda_{A B}=+1\right.$ if $A$ and $B$ are both incoming or outgoing, and $\lambda_{A B}=0$ otherwise) in Eq. (18) are the unitarity phases related to the analytic continuation from unphysical to physical momenta.

The result in Eq. (18) explicitly shows that the soft limit of the one-loop amplitudes is process-independent, meaning that it does not depend on the momentum and colour flows of the internal partons (including the parton circulating in the loop) in the matrix element. This simple structure has several interesting features that we comment below.

The one-loop soft current is proportional to the structure constants $f_{a b c}$ of the gauge group, and thus it is purely non-abelian. This is in agreement with the absence of higherloop corrections to the soft current in massless QED [25].

Since Eq. (18) is proportional to the factor $\left(p_{i}^{\mu} / p_{i} \cdot q-p_{j}^{\mu} / p_{j} \cdot q\right)$, the one-loop contribution to the soft current is conserved:

$$
q_{\mu} J_{a}^{\mu(1)}(q, \epsilon)=0
$$

Combined with the analogous property at tree level, this guarantees that the soft-gluon factorization formula (15) is manifestly gauge-invariant. 
The double pole $1 / \epsilon^{2}$ in Eq. (18) is the infrared singularity produced by a soft and collinear virtual gluon. To double-pole accuracy, we can use colour conservation (see Eq. (7)) to show that the one-loop current is simply proportional to the tree-level current:

$$
J_{\mu}^{a(1)}(q, \epsilon)\left|\mathcal{M}\left(q, p_{1}, \ldots, p_{m}\right)\right\rangle=-\frac{1}{16 \pi^{2}}\left[\frac{C_{A}}{\epsilon^{2}} J_{\mu}^{a(0)}(q)+\mathcal{O}\left(\frac{1}{\epsilon}\right)\right]\left|\mathcal{M}\left(q, p_{1}, \ldots, p_{m}\right)\right\rangle .
$$

This behaviour is consistent with the known singularity structure of the one-loop amplitudes [26, 27, 28].

Beyond the double-pole approximation, the one-loop current contains [29] two-particle colour correlations. The correlations are induced by the last factor on the right-hand side of Eq. (18). This factor fully embodies the logarithmic dependence on the soft-gluon momentum $q$ and has a simple kinematic interpretation, being related to the transverse component $q_{\perp, i j}$ of the gluon momentum with respect to the longitudinal direction singled out by the momenta $p_{i}$ and $p_{j}$ of the colour-correlated hard partons:

$$
q_{\perp, i j}^{2}=\frac{2\left(p_{i} \cdot q\right)\left(p_{j} \cdot q\right)}{p_{i} \cdot p_{j}}
$$

The derivation of the result in Eq. (18) (see Sect. (1) suggests that multiparticle colour correlations will appear in higher-loop contributions to the soft-gluon current (16). For instance, at two-loop order the soft current contains colour correlations of the type

$$
f_{\text {abe }} f_{\text {ecd }} T_{i}^{b} T_{j}^{c} T_{k}^{d}
$$

between three different hard partons $i, j$ and $k$.

The soft behaviour of the one-loop amplitudes was first investigated in Refs. [8, 12, 14] by using the colour-subamplitude formalism. The explicit expressions of the one-loop softgluon contribution to colour subamplitudes, with $m$ external gluons and with $m$ external gluons plus a $q \bar{q}$ pair, were derived in Refs. [12] and [14], respectively. The reader can straightforwardly check that the result in Eq. (18) agrees with those in Refs. [12, 14] for the particular cases considered therein. The general result in Eq. (18) shows that, although colour and kinematics are deeply entangled in the soft region, the soft limit of the one-loop amplitudes can be factorized in colour-space in a way that is both (relatively) simple and process-independent (in particular, independent of the flavour of the external partons). In particular, this general structure is quite useful to show (see below and Sect. 5) that colour and kinematics can be completely disentangled in the computation of the soft limit of the square of one-loop matrix elements with two or three external QCD partons. The factorization formula (17) can be used to compute the soft limit of the one-loop contribution to the square of the matrix element $\mathcal{M}(q,\{p\})$. Summing over the polarizations of the

\footnotetext{
IIn the following the dependence of the matrix element on the momenta $p_{1}, \ldots, p_{m}$ of the hard partons is denoted by $\{p\}$.
} 
soft gluon and using Eqs. (8) and (17), we have

$$
\begin{aligned}
& \left\langle\mathcal{M}^{(0)}(q,\{p\}) \mid \mathcal{M}^{(1)}(q,\{p\})\right\rangle+\text { c.c. } \simeq-\left(g_{S} \mu^{\epsilon}\right)^{2} \\
& \cdot\left\{\left[\left\langle\mathcal{M}^{(0)}(\{p\})\left|\boldsymbol{J}_{\mu}^{(0)}(q) \cdot \boldsymbol{J}^{\mu(0)}(q)\right| \mathcal{M}^{(1)}(\{p\})\right\rangle+\text { c.c. }\right]\right. \\
& \left.+\left(g_{S} \mu^{\epsilon}\right)^{2}\left[\left\langle\mathcal{M}^{(0)}(\{p\})\left|\boldsymbol{J}_{\mu}^{(0)}(q) \cdot \boldsymbol{J}^{\mu(1)}(q, \epsilon)\right| \mathcal{M}^{(0)}(\{p\})\right\rangle+\text { c.c. }\right]\right\},
\end{aligned}
$$

where c.c. denotes the complex conjugate.

The first term on the right-hand side, which can be evaluated by using the expression (9) of the tree-level gluon current, has the same structure as Eq. (12):

$$
\left\langle\mathcal{M}^{(0)}(\{p\})\left|\boldsymbol{J}_{\mu}^{(0)}(q) \cdot \boldsymbol{J}^{\mu(0)}(q)\right| \mathcal{M}^{(1)}(\{p\})\right\rangle+\text { c.c. }=2 \sum_{i, j=1}^{m} \mathcal{S}_{i j}(q)\left|\mathcal{M}_{(i, j)}^{(1)}(\{p\})\right|^{2}
$$

where $\mathcal{S}_{i j}(q)$ is the eikonal function in Eq. (13). In particular, the two-particle colour correlations on the right-hand side are completely analogous to those at tree level (see Eqs. (12) and (14)) and have been taken into account by defining the colour-correlated one-loop amplitude

$$
\left|\mathcal{M}_{(i, j)}^{(1)}(\{p\})\right|^{2} \equiv\left\langle\mathcal{M}^{(0)}(\{p\})\left|\boldsymbol{T}_{i} \cdot \boldsymbol{T}_{j}\right| \mathcal{M}^{(1)}(\{p\})\right\rangle+\text { c.c. } .
$$

Using expression (18) for the one-loop contribution to the soft-gluon current, the second term on the right-hand side of Eq. (23) can be written as

$$
\begin{aligned}
\left\langle\mathcal{M}^{(0)}(\{p\})\right| \boldsymbol{J}_{\mu}^{(0)}(q) \cdot & \boldsymbol{J}^{\mu(1)}(q, \epsilon)\left|\mathcal{M}^{(0)}(\{p\})\right\rangle+\text { c.c. }=-\frac{1}{4 \pi^{2}} \frac{(4 \pi)^{\epsilon}}{\epsilon^{2}} \frac{\Gamma^{3}(1-\epsilon) \Gamma^{2}(1+\epsilon)}{\Gamma(1-2 \epsilon)} \\
\cdot & \left\{C_{A} \cos (\pi \epsilon) \sum_{i, j}^{\prime}\left[\mathcal{S}_{i j}(q)\right]^{1+\epsilon}\left|\mathcal{M}_{(i, j)}^{(0)}(\{p\})\right|^{2}\right. \\
+ & \left.2 \sin (\pi \epsilon) \sum_{i, j, k}^{\prime} \mathcal{S}_{k i}(q)\left[\mathcal{S}_{i j}(q)\right]^{\epsilon}\left(\lambda_{i j}-\lambda_{i q}-\lambda_{j q}\right)\left|\mathcal{M}_{(k, i, j)}^{(0)}(\{p\})\right|^{2}\right\},
\end{aligned}
$$

where we have used $\left(\lambda_{i j}-\lambda_{i q}-\lambda_{j q}\right)^{2}=1$ for any possible configuration of incoming and outgoing momenta, and the notation $\sum^{\prime}$ stands for the sum over the different values of the indices $(i \neq j, j \neq k, k \neq i)$.

The one-loop contribution on the right-hand side of Eq. (26) contains two terms. The first term only involves colour correlations between two hard partons, which are taken into account by the colour-correlated tree-amplitude $\mathcal{M}_{(i, j)}^{(0)}(\{p\})$ defined in Eq. (14). Thus, apart from an overall $\epsilon$-dependent factor, its effect simply amounts to the rescaling $\mathcal{S}_{i j}(q) \rightarrow$ $\left[\mathcal{S}_{i j}(q)\right]^{1+\epsilon}$ in the tree-level factorization formula $([12)$. The second term instead has a different structure, because it leads to colour correlations between three different hard partons. These are included in the three-parton correlated tree-amplitude:

$$
\left|\mathcal{M}_{(k, i, j)}^{(0)}(\{p\})\right|^{2} \equiv f_{a b c}\left\langle\mathcal{M}^{(0)}(\{p\})\left|T_{k}^{a} T_{i}^{b} T_{j}^{c}\right| \mathcal{M}^{(0)}(\{p\})\right\rangle .
$$


Note that the second term contributes only when there are four or more hard partons, because in the case of three partons colour conservation (see Eq. (7)) can be used to show that the three-parton correlations vanish:

$$
f_{a b c} T_{1}^{a} T_{2}^{b} T_{3}^{c}\left|\mathcal{M}^{(0)}\left(p_{1}, p_{2}, p_{3}\right)\right\rangle=-f_{a b c} T_{1}^{a} T_{2}^{b}\left(T_{1}^{c}+T_{2}^{c}\right)\left|\mathcal{M}^{(0)}\left(p_{1}, p_{2}, p_{3}\right)\right\rangle=0 .
$$

The absence of these correlations extremely simplifies the structure of the soft limit of the squared matrix elements with two or three hard partons. As a matter of fact, in these cases any product $\boldsymbol{T}_{i} \cdot \boldsymbol{T}_{j}$ can be expressed as a linear combination of Casimir operators (see Sect. 5). Therefore, the colour algebra can explicitly be carried out and the soft limit of $|\mathcal{M}(q,\{p\})|^{2}$ is directly proportional to $|\mathcal{M}(\{p\})|^{2}$ at one-loop accuracy. The corresponding explicit expressions are presented in Sect. 5 .

Note also that in the limit $\epsilon \rightarrow 0$ the three-particle correlation contribution to Eq. (26) is at most as singular as $1 / \epsilon$. More precisely, it gives rise to single poles $1 / \epsilon$ only when there are two or more incoming partons. In fact, in the case of an outgoing soft gluon $q$, we have $\lambda_{i j}-\lambda_{i q}-\lambda_{j q}=+1$ when $i$ and $j$ are both incoming momenta and $\lambda_{i j}-\lambda_{i q}-\lambda_{j q}=-1$ otherwise. Therefore, we can rewrite the second term in the curly bracket of Eq. (26) as

$$
\begin{aligned}
& +4 \sin (\pi \epsilon) \sum_{i, j(\mathrm{in}), k}{ }^{\prime} \mathcal{S}_{k i}(q)\left[\mathcal{S}_{i j}(q)\right]^{\epsilon} f_{a b c}\left\langle\mathcal{M}^{(0)}(\{p\})\left|T_{k}^{a} T_{i}^{b} T_{j}^{c}\right| \mathcal{M}^{(0)}(\{p\})\right\rangle \\
& -2 \sin (\pi \epsilon) \sum_{i, j, k}^{\prime} \mathcal{S}_{k i}(q)\left[\mathcal{S}_{i j}(q)\right]^{\epsilon} f_{a b c}\left\langle\mathcal{M}^{(0)}(\{p\})\left|T_{k}^{a} T_{i}^{b} T_{j}^{c}\right| \mathcal{M}^{(0)}(\{p\})\right\rangle,
\end{aligned}
$$

where the sum $\sum_{i, j(\mathrm{in}), k}^{\prime}$ denotes the restriction of $\sum_{i, j, k}^{\prime}$ to the indices $i, j$ of the incoming partons. Then, it is easy to show that the second term in Eq. (29) is of $\mathcal{O}\left(\epsilon^{2}\right)$ in the limit $\epsilon \rightarrow 0$ :

$$
\begin{aligned}
& -2 \sin (\pi \epsilon) \sum_{i, j, k}^{\prime} \mathcal{S}_{k i}(q)\left[\mathcal{S}_{i j}(q)\right]^{\epsilon} f_{a b c}\left\langle\mathcal{M}^{(0)}(\{p\})\left|T_{k}^{a} T_{i}^{b} T_{j}^{c}\right| \mathcal{M}^{(0)}(\{p\})\right\rangle= \\
& -2 \pi \epsilon \sum_{i, j, k}^{\prime} \mathcal{S}_{k i}(q) f_{a b c}\left\langle\mathcal{M}^{(0)}(\{p\})\left|T_{k}^{a} T_{i}^{b} T_{j}^{c}\right| \mathcal{M}^{(0)}(\{p\})\right\rangle+\mathcal{O}\left(\epsilon^{2}\right)= \\
& +2 \pi \epsilon \sum_{i, k}^{\prime} \mathcal{S}_{k i}(q) f_{a b c}\left\langle\mathcal{M}^{(0)}(\{p\})\left|T_{k}^{a} T_{i}^{b}\left(T_{i}^{c}+T_{k}^{c}\right)\right| \mathcal{M}^{(0)}(\{p\})\right\rangle+\mathcal{O}\left(\epsilon^{2}\right)=\mathcal{O}\left(\epsilon^{2}\right) .
\end{aligned}
$$

Here we have set $\left[\mathcal{S}_{i j}(q)\right]^{\epsilon} \rightarrow 1$. Then we have used colour conservation, $\sum_{j \neq i, k} T_{j}^{c}=$ $-\left(T_{i}^{c}+T_{k}^{c}\right)$, and the identity $f_{a b c} T_{k}^{a} T_{i}^{b}\left(T_{i}^{c}+T_{k}^{c}\right)=0$ for $i \neq k$.

\section{Proof of factorization and calculation of the one-loop current}

In this section we derive the factorization formula (15) at one-loop order and we explicitly compute the one-loop contribution to the soft-gluon current.

To simplify the analysis, it is convenient to work in a gauge with only physical gluon polarizations. We use the axial gauge $n \cdot A=0$ with a light-like $\left(n^{2}=0\right)$ gauge vector 
$n^{\mu}$. The polarization vectors $\varepsilon^{\mu}(k)$ of a gluon with momentum $k$ thus fulfil the relations $n \cdot \varepsilon(k)=0$ and $k \cdot \varepsilon(k) \propto k^{2}$. The sum over the gluon polarizations leads to the polarization tensor $d^{\mu \nu}$ :

$$
d^{\mu \nu}(k)=\sum_{\text {pol. }} \varepsilon^{\mu}(k) \varepsilon^{\nu}(k)=-g^{\mu \nu}+\frac{k^{\mu} n^{\nu}+n^{\mu} k^{\nu}}{n \cdot k} .
$$

The expression on the right-hand side corresponds to a dimensional-regularization scheme with $d-2=2-2 \epsilon$ gluon polarizations. For the sake of definiteness we use this explicit expression in all the intermediate steps of the calculation. However, we shall show that the final results are regularization-scheme independent by pointing out in which steps the scheme dependence might arise.

\subsection{Proof of factorization}

We study the soft behaviour of one-loop matrix elements by using the eikonal approximation and the soft-gluon insertion rulesth recalled above Eq. (10). For this purpose, it is useful to decompose the one-loop matrix element $\mathcal{M}^{(1)}$ in three contributions,

$$
\mathcal{M}^{(1)}=\mathcal{M}_{\text {hard }}^{(1)}+\mathcal{M}_{\text {coll }}^{(1)}+\mathcal{M}_{\text {soft }}^{(1)}
$$

which respectively represent the kinematic regions where the momentum $k$ circulating in the loop is

- hard: its momentum components are of the same order as those of the hard external momenta $p$,

- collinear: $k$ is parallel to one of the hard external momenta,

- soft: its momentum components are much smaller than those of the hard external momenta $p$.

For the purpose of the following discussion, we recall that the soft virtual behaviour of the one-loop matrix element $\mathcal{M}^{(1)}(\{p\})$ can be computed by using the soft-gluon insertion rules. The soft virtual gluon of momentum $k$ is inserted (emitted and reabsorbed) on all the external legs (Fig. 1) of the tree-level amplitude $\mathcal{M}^{(0)}(\{p\})$, leading to the expression [26] 29, 31]:

$$
\left|\mathcal{M}_{\text {soft }}^{(1)}(\{p\})\right\rangle=\frac{1}{2} g_{\mathrm{S}}^{2} \mu^{2 \epsilon} \int \frac{d^{d} k}{(2 \pi)^{d}} \frac{i}{k^{2}+i 0}\left[J_{\mu}^{a(0)}(k)\right]^{\dagger} J^{\mu a(0)}(k)\left|\mathcal{M}^{(0)}(\{p\})\right\rangle,
$$

where $J_{\mu}^{a(0)}(k)$ is the (tree-level) soft-gluon current in Eq. (9).

\footnotetext{
"We use the eikonal approximation both for real and for virtual soft gluons. In the case of virtual gluons, the eikonal approximation is not always justified for each single Feynman diagram. Nonetheless, it is valid for any gauge-invariant set of Feynman diagrams, such as those computed in this section. This can be shown 30] by simply using time-ordered perturbation theory, where the eikonal approximation is valid on a graph-by-graph basis.
} 


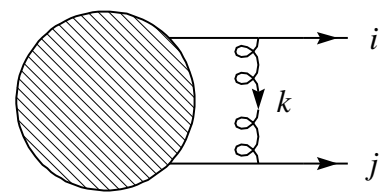

(a)

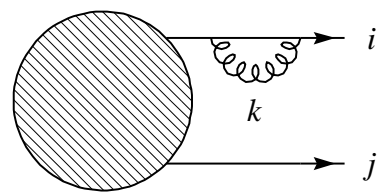

(b)

Figure 1: Feynman diagrams that contribute to the soft behaviour of the one-loop amplitude. The shaded blob denotes the tree-level amplitude and the virtual gluon with soft momentum $k$ can either (a) connect two different external legs $i$ and $j$ or $(b)$ be emitted and reabsorbed by the same leg.

We now consider the one-loop matrix element $\mathcal{M}^{(1)}(q,\{p\})$ when the momentum $q$ of the external gluon becomes soft. To apply the soft-gluon insertion rules, we perform the decomposition in Eq. (33) and discuss the three different kinematic regions of the virtual momentum $k$ in turn.

When $k$ is in the hard region, all the internal lines in $\mathcal{M}^{(1)}(q,\{p\})$ are highly off-shell. Thus the real soft gluon $q$ can couple only to the external legs and we can factorize its contribution as in the case of tree-level amplitudes. Neglecting terms that are not singular in the soft limit $q \rightarrow 0$, we get

$$
\left\langle a \mid \mathcal{M}_{\text {hard }}^{(1)}(q,\{p\})\right\rangle \simeq g_{S} \mu^{\epsilon} \varepsilon^{\mu}(q) J_{\mu}^{a(0)}(q)\left|\mathcal{M}_{\text {hard }}^{(1)}(\{p\})\right\rangle,
$$

where $J_{\mu}^{a(0)}(q)$ is the soft-gluon current in Eq. (9).

We now consider the region where the loop momentum $k$ is collinear to the momentum of one, say $p_{i}$, of the hard external legs. Since we work in a physical gauge, the only diagram that is not dynamically suppressed [4] is that in which the loop leads to a selfenergy contribution on the external leg $p_{i}$ (e.g. the diagram in Fig. 1 (b)). The soft gluon can then be inserted in this diagram in all possible ways: on the line $p_{i}$ before and after the self-energy contribution and on the self-energy lines themselves. However, we can exploit the colour coherence properties [4] of QCD radiation. Since the loop-momentum $k$ is parallel to $p_{i}$, the soft gluon $q$ cannot distinguish the self-energy lines from a single line with total colour charge $T_{i}^{a}$. The sum of the insertions of $q$ on this diagram is thus insensitive to the presence of the collinear loop momentum and leads (see, for instance, Sect. 3.4 in Ref. [7] for a detailed similar discussion) to the same factor, $T_{i}^{a} p_{i} \cdot \varepsilon(q) / p_{i} \cdot q$, as in the soft-gluon factorization at tree level. Considering the insertions of $q$ on all the other external legs $j \neq i$, we obtain a factorization formula of tree-level type also in the collinear region:

$$
\left\langle a \mid \mathcal{M}_{\text {coll }}^{(1)}(q,\{p\})\right\rangle \simeq g_{S} \mu^{\epsilon} \varepsilon^{\mu}(q) J_{\mu}^{a(0)}(q)\left|\mathcal{M}_{\text {coll }}^{(1)}(\{p\})\right\rangle .
$$

We finally have to deal with the region in which the loop momentum $k$ is carried by a soft gluon E* Unlike the case of the hard and collinear regions, where the one-loop effects

\footnotetext{
** Quarks loops are dynamically suppressed when their momentum become soft. Without loss of infrared accuracy, we thus consider the quark loops as included in the hard or collinear regions.
} 
can be factorized with respect to the tree-level current $J_{\mu}^{a(0)}(q)$ (see Eqs. (35) and (36)), new 'non-factorizable' contributions appear when the loop momentum is soft. To single out these new contributions, we write the following identity:

$$
\begin{aligned}
\left|\mathcal{M}_{\text {soft }}^{(1)}(q,\{p\})\right\rangle & =g_{S} \mu^{\epsilon} \varepsilon^{\mu}(q) \boldsymbol{J}_{\mu}^{(0)}(q)\left|\mathcal{M}_{\text {soft }}^{(1)}(\{p\})\right\rangle \\
& +\left(\left|\mathcal{M}_{\text {soft }}^{(1)}(q,\{p\})\right\rangle-g_{S} \mu^{\epsilon} \varepsilon^{\mu}(q) \boldsymbol{J}_{\mu}^{(0)}(q)\left|\mathcal{M}_{\text {soft }}^{(1)}(\{p\})\right\rangle\right)
\end{aligned}
$$

where we have added and subtracted the 'factorized' contribution. Then we combine the contributions from the hard, collinear and soft regions by adding Eqs. (35), (36) and (37), and we obtain

$$
\begin{aligned}
\left|\mathcal{M}^{(1)}(q,\{p\})\right\rangle & =g_{S} \mu^{\epsilon} \varepsilon^{\mu}(q) \boldsymbol{J}_{\mu}^{(0)}(q)\left|\mathcal{M}^{(1)}(\{p\})\right\rangle \\
& +\left(\left|\mathcal{M}_{\text {soft }}^{(1)}(q,\{p\})\right\rangle-g_{S} \mu^{\epsilon} \varepsilon^{\mu}(q) \boldsymbol{J}_{\mu}^{(0)}(q)\left|\mathcal{M}_{\text {soft }}^{(1)}(\{p\})\right\rangle\right) .
\end{aligned}
$$

The first term on the right-hand side of Eq. (37) together with the contributions from Eqs. (35) and (36) have reconstructed the first term on the right-hand side of Eq. (38), which is exactly the first term on the right-hand side of the factorization formula (17). What remains to be done to prove the factorization formula is to relate the second term on the right-hand side of Eq. (17) with the contribution in the round bracket of Eq. (38).

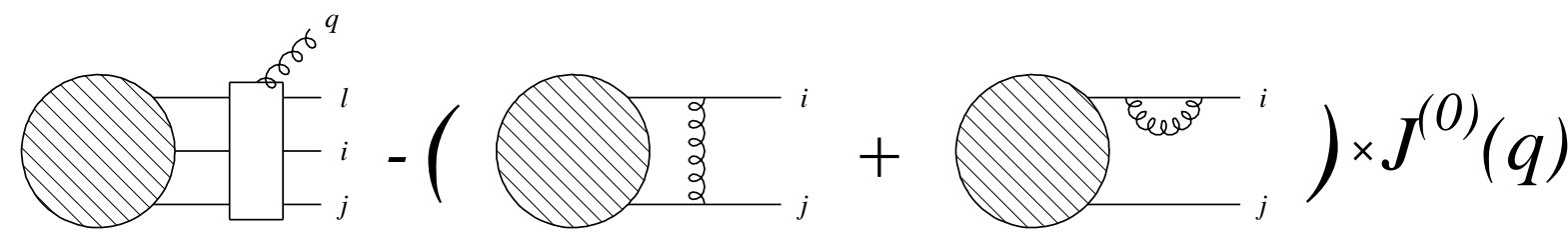

Figure 2: Graphs that contribute to the one-loop soft current.

For this purpose, we first note that when the real gluon $q$ and the virtual gluon $k$ are both soft, they can couple only to the external hard lines. In the corresponding Feynman diagrams, which are schematically represented by the first graph in Fig. 2, the tree-level amplitude $\mathcal{M}^{(0)}(\{p\})$ is factorized in the soft limit. We can write:

$$
\left|\mathcal{M}_{\text {soft }}^{(1)}(q,\{p\})\right\rangle \simeq\left(g_{S} \mu^{\epsilon}\right)^{3} \varepsilon^{\mu}(q) \boldsymbol{K}_{\mu}^{(1)}(q, \epsilon)\left|\mathcal{M}^{(0)}(\{p\})\right\rangle
$$

where the kernel $\boldsymbol{K}^{(1)}$ (represented by the box in Fig. 2) denotes all the soft-gluon insertions of $q$ and $k$ on the hard-momentum lines. Then, we note that $\mathcal{M}^{(0)}(\{p\})$ is factorized also in the expression (34) for $\mathcal{M}_{\text {soft }}^{(1)}(\{p\})$. Therefore, the contribution in the round bracket of Eq. (B8) can be recast in the form of the second term on the right-hand side of the factorization formula (17). Moreover, using Eqs. (39) and (34), we obtain the following explicit representation of the one-loop contribution $\boldsymbol{J}^{(1)}$ to the soft-gluon current (Fig. 2):

$\varepsilon^{\mu}(q) \boldsymbol{J}_{\mu}^{(1)}(q, \epsilon)=\varepsilon^{\mu}(q)\left\{\boldsymbol{K}_{\mu}^{(1)}(q, \epsilon)-\boldsymbol{J}_{\mu}^{(0)}(q) \frac{1}{2} \int \frac{d^{d} k}{(2 \pi)^{d}} \frac{i}{k^{2}+i 0}\left[\boldsymbol{J}_{\nu}^{(0)}(k)\right]^{\dagger} \cdot \boldsymbol{J}^{\nu(0)}(k)\right\}$. 


\subsection{Calculation of the one-loop current}

We now proceed to the explicit calculation of the one-loop soft current $\boldsymbol{J}^{(1)}$. Using the eikonal approximation, we have to evaluate the Feynman diagrams of the kernel $\boldsymbol{K}^{(1)}$ and to subtract those corresponding to the second term on the right-hand side of Eq. (40). We divide the diagrams in two classes: $(A)$ the diagrams that involve interactions with a single

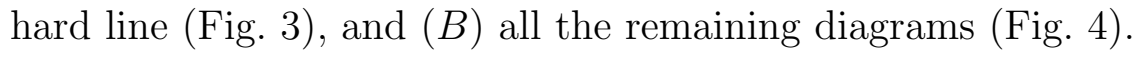

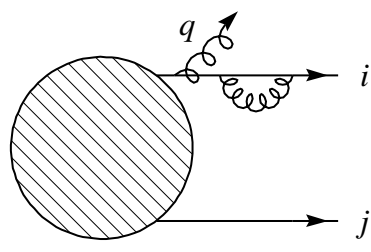

(a)

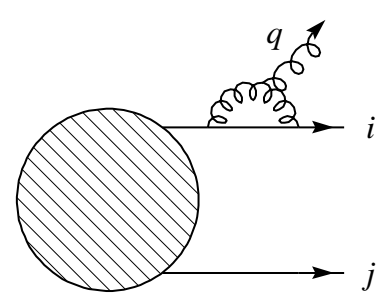

(d)

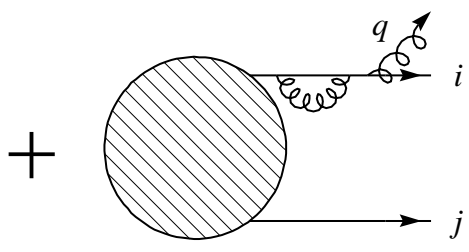

(b)

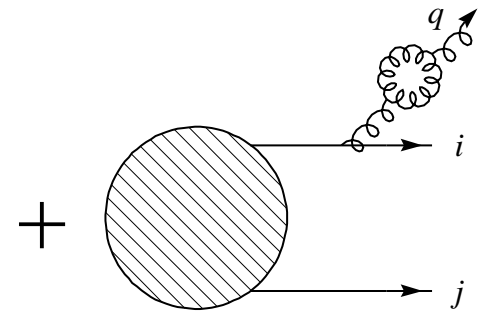

(e)

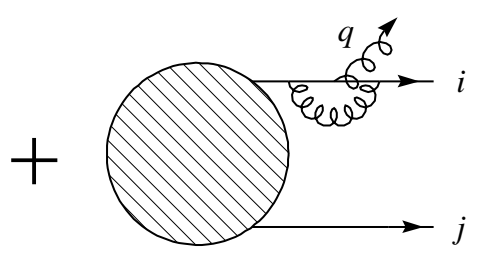

(c)

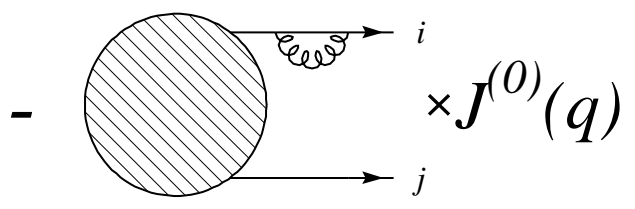

(f)

Figure 3: Feynman diagrams that depend on a single hard momentum.

We first consider class $(B)$. The diagrams $(a),(b),(c)$ and $(d)$ in Fig. 1 come from the kernel $\boldsymbol{K}^{(1)}$, while Fig. $1(e)$ represents the corresponding subtractions. We see that within this class there are diagrams that involve interactions between three different external lines $i, j$ and $l$. These diagrams cancel. Indeed, the diagram in Fig. $1(d)$ is exactly cancelled by that (e)-contribution in which $q$ is emitted by the line $l$ (the sum over the emissions of $q$ is included in the factor $\left.\boldsymbol{J}^{(0)}(q)\right)$. Thus the only non-vanishing terms coming from class $(B)$ are those that involve interactions between two external lines, namely the diagrams $(a),(b),(c)$ and the corresponding subtractions in $(e)$. The diagrams $(a),(c)$ and the subtractions in (e) are very similar: they can be combined in a simple way, because they only differ by the momentum and colour flow along the hard line $i$. The different factors coming from the line $i$ in the diagrams $(c)$ and $(e)$ give

$$
+T_{i}^{a} T_{i}^{b} \frac{1}{p_{i} q+i 0} \frac{1}{p_{i}(k+q)+i 0}-T_{i}^{a} T_{i}^{b} \frac{1}{p_{i} q+i 0} \frac{1}{p_{i} k+i 0}
$$

while the corresponding factor from the diagram $(a)$ is

$$
+T_{i}^{b} T_{i}^{a} \frac{1}{p_{i} k+i 0} \frac{1}{p_{i}(k+q)+i 0} .
$$

Decomposing the colour factor in Eq. (42) in its non-abelian and abelian components, $T_{i}^{b} T_{i}^{a}=i f_{b a c} T_{i}^{c}+T_{i}^{a} T_{i}^{b}$, and adding Eq. (41), we see that the abelian component $T_{i}^{a} T_{i}^{b}$ of the 


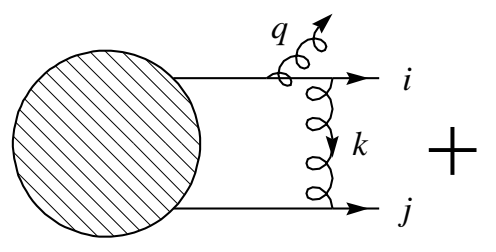

(a)

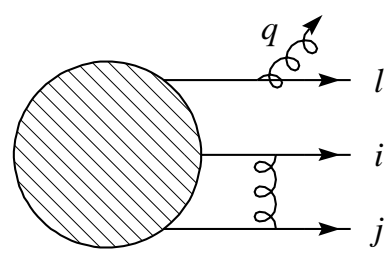

(d)

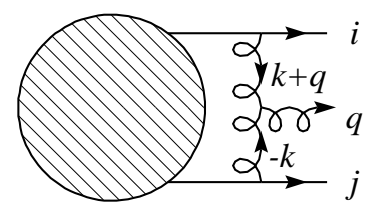

(b)

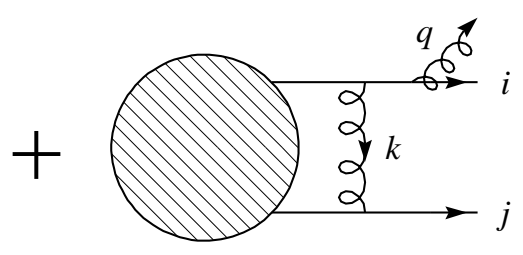

(c)

Figure 4: Feynman diagrams that depend on two or three different hard momenta.

diagram $(a)$ exactly cancels the diagrams $(c)$ and $(e)$. In conclusion, the total contribution to the one-loop current from class $(B)$ simply amounts to computing the diagram $(b)$, which is non-abelian, and the non-abelian part of the diagram $(a)$, which is obtained by the replacement $T_{j}^{b} T_{i}^{b} T_{i}^{a} \rightarrow i f_{b a c} T_{j}^{b} T_{i}^{c}$ (b is the colour index of the virtual gluon) in its overall colour factor.

The calculation of the diagrams in Fig. $1(a)$ and $(b)$ is quite simple (see below). Then we have to add the diagrams of class $(A)$ (Fig. 3). An argument similar to that in Eqs. (41) and (42) can be used to show that the abelian parts of these diagrams cancel $\square$. Thus, only their non-abelian part has to be evaluated. Although straightforward, this calculation is quite cumbersome, in particular because it has to be carried out in the axial gauge $n \cdot A=0$. This cumbersome calculation can be short-circuited by exploiting gauge invariance.

We first recall that the non-vanishing contributions from class $(B)$ depend on the momenta and charges of (at most) two hard partons. Since the diagrams of class $(A)$ involve interactions with a single hard line, we can split the one-loop soft current in two terms, $J_{1 P}$ and $J_{2 P}$, that respectively denote the contributions that depend on one and two hard momenta:

$$
J_{\mu}^{a(1)}(q, \epsilon)=J_{\mu ; 1 P}^{a(1)}(q, \epsilon)+J_{\mu ; 2 P}^{a(1)}(q, \epsilon) .
$$

All the diagrams of class $(A)$ are included in $J_{1 P}$. Those of class $(B)$ contribute to $J_{2 P}$ and (because of possible cancellations in the dependence of one hard momentum) to $J_{1 P}$. We shall explicitly compute $J_{2 P}$ and show that it is gauge-invariant. Thus $J_{1 P}$ has to be gauge invariant as well, that is, it cannot depend on the gauge vector $n^{\nu}$. Power counting and the fact that the one-loop current is non-abelian are then sufficient to determine $J_{1 P}$,

\footnotetext{
${ }^{\dagger \dagger}$ More precisely, the abelian diagrams in Figs. $3(a)$ and $(b)$ are cancelled by the abelian part of the diagram in Fig. $3(c)$.
} 
apart from an overall factor $f^{(1)}(\epsilon)$ that can only depends on $\epsilon$ :

$$
J_{\mu ; 1 P}^{a(1)}(q, \epsilon)=C_{A} f^{(1)}(\epsilon) \sum_{i=1}^{m}\left(p_{i} \cdot q\right)^{-\epsilon} \frac{p_{i \mu}}{p_{i} \cdot q} T_{i}^{a} .
$$

This overall factor can then be obtained by imposing that the soft-gluon factorization formula be gauge-invariant (with respect to the polarizations of the real gluon $q$ ) or, equivalently, that the complete one-loop current in Eq. (43) be conserved. This leads to the constraint

$$
q^{\mu} J_{\mu}^{a(1)}(q, \epsilon)=q^{\mu} J_{\mu ; 2 P}^{a(1)}(q, \epsilon)+C_{A} f^{(1)}(\epsilon) \sum_{i=1}^{m}\left(p_{i} \cdot q\right)^{-\epsilon} T_{i}^{a}=0
$$

which can be used to get $f^{(1)}(\epsilon)$ (and, hence, $J_{\mu ; 1 P}^{a(1)}$ ) from the two-parton contribution $J_{\mu ; 2 P}^{a(1)}$. In particular, the calculation of the diagrams of class $(A)$ can be avoided.

We anticipate that our explicit calculation of the two-parton contribution $J_{\mu ; 2 P}^{a(1)}$ gives $q^{\mu} J_{\mu ; 2 P}^{a(1)}(q, \epsilon)=0$. Thus the single-parton contribution $J_{\mu ; 1 P}^{a(1)}$ vanishes. Note that this does not mean that the diagrams of class $(A)$ give a vanishing contribution, but rather that their contribution is cancelled by the terms of class $(B)$ that depend on a single hard momentum.

We can now complete our calculation of the one-loop current by explicitly computing the non-abelian part of the diagrams in Figs. $1(a)$ and (b), and, more precisely, their contribution to $J_{\mu ; 2 P}^{a(1)}$. For the sake of definiteness, we write down the diagrams for the case in which all external momenta are outgoing. The diagram in Fig. $4(a)$ depends on the polarization tensor $d(k)$ of the virtual gluon. Using the expression in Eq. (32) and performing the Lorentz algebra of the spin numerator, the non-abelian part of the diagram in Fig. $1(a)$ gives

$$
\begin{aligned}
f_{a b c} \sum_{i \neq j} T_{i}^{c} T_{j}^{b} \int \frac{d^{d} k}{(2 \pi)^{d}} \frac{1}{\left(k^{2}+i 0\right)\left(p_{j} \cdot k-i 0\right)} \frac{p_{i} \cdot \varepsilon(q)}{p_{i} \cdot(k+q)+i 0} \\
\cdot \frac{1}{p_{i} \cdot k+i 0}\left(p_{i} \cdot p_{j}-p_{j} \cdot k \frac{p_{i} \cdot n}{k \cdot n}-p_{i} \cdot k \frac{p_{j} \cdot n}{k \cdot n}\right) .
\end{aligned}
$$

The diagram in Fig. $1(b)$ depends on the polarization tensors $d(k)$ and $d(k+q)$ of the virtual-gluon lines. Performing the Lorentz algebra of the spin numerator and exploiting the symmetry of the integrand under the transformation $i \leftrightarrow j, k \leftrightarrow-(k+q)$, the diagram in Fig. 1 (b) gives

$$
\begin{aligned}
& f_{a b c} \sum_{i \neq j} T_{i}^{c} T_{j}^{b} \int \frac{d^{d} k}{(2 \pi)^{d}} \frac{1}{\left(k^{2}+i 0\right)\left(p_{j} \cdot k-i 0\right)} \frac{1}{p_{i} \cdot(k+q)+i 0} \frac{1}{(k+q)^{2}+i 0} \varepsilon_{\mu}(q) \\
& \cdot\left[k^{\mu} p_{i} \cdot p_{j}-p_{i}^{\mu} p_{j} \cdot(k+2 q)-p_{j} \cdot k\left(2 k^{\mu} \frac{p_{i} \cdot n}{k \cdot n}-p_{i}^{\mu} \frac{(k+2 q) \cdot n}{k \cdot n}\right)+p_{i}^{\mu}(k+q)^{2} \frac{p_{j} \cdot n}{k \cdot n}\right] .
\end{aligned}
$$

$\ddagger \ddagger$ Note that we use the eikonal approximation for the vertices and propagators of the hard-momentum lines $i$ and $j$, while gluon propagators and the three-gluon vertex must be treated exactly. 
By inspection of Eqs. (46) and (47), we see that the last term in the round bracket of Eq. (46) cancels the last term in the square bracket of Eq. (47). The remaining $n$-dependence of the integrands is proportional to $p_{j} \cdot k$. This factor cancels the fermion propagator $1 /\left(p_{j} \cdot k-i 0\right)$, thus leaving a contribution that, although $n$-dependent, depends on a single hard momentum $p_{i}$. This term can then be included, together with the class $(A)$ diagrams, in the single-particle contribution $J_{\mu ; 1 P}^{(1)}(q, \epsilon)$ to the one-loop current. We conclude that (the contribution of Eqs. (46) and (47) to) the two-particle current $J_{\mu ; 2 P}^{(1)}(q, \epsilon)$ is explicitly independent of the gauge vector $n^{\mu}$. Note also that $n$-dependence cancels at the integrand level, and thus, the cancellation is completely insensitive to the actual prescription [32] used to regularize the gauge pole $1 /(n \cdot k)$ of the gluon polarization tensor (32).

Although the expression (32), which we have used for the polarization tensor, corresponds to $d-2=2-2 \epsilon$ helicity states for the gluon, our calculation also allows us to discuss the (in)dependence on the dimensional-regularization scheme. Other regularization schemes [20, 21] use 2 helicity states for the gluon. At one-loop order, the difference eventually amounts [22, 23] to set $\epsilon=0$ in the integrands after having performed the Lorentz algebra of the spin numerators. Since the integrands of Eqs. (46) and (47) have no explicit $\epsilon$-dependence, our result for $\varepsilon(q) \cdot J_{2 P}^{(1)}$ (and hence for the complete current $\varepsilon(q) \cdot J^{(1)}$ ) does not depend on the scheme used to implement dimensional regularization.

We proceed to the computation of $\varepsilon(q) \cdot J_{2 P}^{(1)}$ by adding the two-particle contributions from Eqs. (46) and (47). As for Eq. (47), the term proportional to $k^{\mu}$ can be reduced to scalar integrals. Then using $q \cdot \varepsilon(q)=0$ and exploiting the symmetry with respect to the transformation $i \leftrightarrow j, k \leftrightarrow-(k+q)$, we obtain

$$
\begin{aligned}
\varepsilon(q) \cdot J_{2 P}^{a(1)}(q, \epsilon) & =f_{a b c} \sum_{i \neq j} T_{i}^{c} T_{j}^{b} \int \frac{d^{d} k}{(2 \pi)^{d}} \frac{p_{i} \cdot \varepsilon(q)}{p_{i} \cdot(k+q)+i 0} \frac{1}{\left(k^{2}+i 0\right)\left(p_{j} \cdot k-i 0\right)} \\
& \cdot\left[\frac{p_{i} \cdot p_{j}}{p_{i} \cdot k+i 0}+\frac{1}{(k+q)^{2}+i 0}\left(\frac{p_{i} \cdot p_{j}}{p_{i} \cdot q} k \cdot q-2 p_{j} \cdot q-\frac{p_{j} \cdot q}{p_{i} \cdot q} p_{i} \cdot k\right)\right] .
\end{aligned}
$$

The ultraviolet-finite integral can be easily performed in the collinear frame in which $p_{i}$ and $p_{j}$ are directed along the '+' and '-' light-cone directions, respectively. In this frame, the integration over the $k_{+}$-complex plane receives contributions from the pole $1 /\left(p_{j} \cdot k-i 0\right)$ in the fermion propagators and from the poles in the gluon propagators. Closing the integration contour on the lower half-plane, and using the residue theorem, we can select only the gluon poles, which amounts to the following replacements in Eq. (48):

$$
\frac{1}{k^{2}+i 0} \rightarrow-2 \pi i \delta_{+}\left(k^{2}\right), \quad \frac{1}{(k+q)^{2}+i 0} \rightarrow-2 \pi i \delta_{+}\left((k+q)^{2}\right)
$$

Therefore Eq. (48) can be expressed as follows

$$
\begin{aligned}
\varepsilon(q) \cdot J_{2 P}^{a(1)}(q, \epsilon) & =-\frac{i}{2} f_{a b c} \sum_{i \neq j} T_{i}^{c} T_{j}^{b} \frac{p_{i} \cdot \varepsilon(q)}{p_{i} \cdot q}\left[I\left(p_{i}, p_{j} ; p_{i} ; 2 p_{i} \cdot q\right)+I\left(p_{i}, p_{j} ; p_{j} ;-2 p_{j} \cdot q-i 0\right)\right. \\
& \left.-I\left(q, p_{j} ; p_{j} ;-2 p_{j} \cdot q-i 0\right)-I\left(p_{j}, q ; p_{i} ; 2 p_{i} \cdot q\right)-I\left(p_{i}, q ; p_{j} ;-2 p_{j} \cdot q-i 0\right)\right],
\end{aligned}
$$


in terms of the master integral

$$
I(p, \bar{p} ; r ; s)=\int \frac{d^{d} k}{(2 \pi)^{d-1}} \delta_{+}\left(k^{2}\right) \frac{p \cdot \bar{p}}{(p \cdot k)(\bar{p} \cdot k)} \frac{s}{2 r \cdot k+s},
$$

which depends on the light-like momenta $p^{\mu}, \bar{p}^{\mu}$ and $r^{\mu}$ and on the complex scalar $s$. The evaluation of the $d$-dimensional integration in Eq. (51) over the on-shell gluon momentum $k$ is straightforward and gives

$$
I(p, \bar{p} ; r ; s)=\frac{1}{8 \pi^{2}}\left[\frac{8 \pi(p \cdot r)(\bar{p} \cdot r)}{s^{2}(p \cdot \bar{p})}\right]^{\epsilon} \frac{1}{\epsilon^{2}} \Gamma^{2}(1-\epsilon) \Gamma(1+\epsilon) \Gamma(1+2 \epsilon) .
$$

Since $I(p, \bar{p} ; r ; s)$ vanishes when $r=p$ or $r=\bar{p}$, inserting Eq. (52) in Eq. (50), we finally obtain

$$
\begin{aligned}
\varepsilon(q) \cdot J_{2 P}^{a(1)}(q, \epsilon)= & \frac{1}{16 \pi^{2}} i f_{a b c} \sum_{i \neq j} T_{i}^{c} T_{j}^{b} \frac{p_{i} \cdot \varepsilon(q)}{p_{i} \cdot q}\left(\frac{4 \pi p_{i} \cdot p_{j}}{2 p_{i} \cdot q p_{j} \cdot q}\right)^{\epsilon} \\
& \cdot \frac{1}{\epsilon^{2}} \Gamma^{2}(1-\epsilon) \Gamma(1+\epsilon) \Gamma(1+2 \epsilon)\left[1+\left(\frac{1}{e^{-2 \pi i}}\right)^{\epsilon}\right] \\
= & -\frac{1}{16 \pi^{2}} i f_{a b c} \sum_{i \neq j} T_{i}^{b} T_{j}^{c} \varepsilon_{\mu}(q)\left(\frac{p_{i}^{\mu}}{p_{i} \cdot q}-\frac{p_{j}^{\mu}}{p_{j} \cdot q}\right)\left(\frac{4 \pi p_{i} \cdot p_{j}}{2 p_{i} \cdot q p_{j} \cdot q e^{-i \pi}}\right)^{\epsilon} \\
& \cdot \frac{1}{\epsilon^{2}} \frac{\Gamma^{3}(1-\epsilon) \Gamma^{2}(1+\epsilon)}{\Gamma(1-2 \epsilon)}
\end{aligned}
$$

where we have used the identity

$$
\cos (\pi \epsilon)=\frac{\Gamma(1+\epsilon) \Gamma(1-\epsilon)}{\Gamma(1+2 \epsilon) \Gamma(1-2 \epsilon)},
$$

and the symmetry under the replacement $i \leftrightarrow j$.

Note that the current in Eq. (53) is conserved, $q^{\mu} J_{\mu ; 2 P}^{a(1)}(q, \epsilon)=0$. Therefore, as discussed below Eq. (45), the single-particle term $J_{\mu ; 1 P}^{a(1)}$ vanishes and Eq. (53) gives the complete oneloop contribution to the soft-gluon current. We conclude that we have explicitly derived the result in Eq. (18) in the case of outgoing parton momenta $p_{i}, p_{j}$ (when $\lambda_{i j}=\lambda_{i q}=\lambda_{j q}=$ +1 ). The completely general result in Eq. (18) is straightforwardly obtained by crossing $\left(p_{i} \rightarrow-p_{i}\right.$ and/or $\left.p_{j} \rightarrow-p_{j}\right)$ the parton momenta in Eq. (48) and repeating the steps that lead to Eq. (53).

\section{$5 \quad$ Processes with two and three partons and $e^{+} e^{-} \rightarrow 3$ jets at NNLO}

In general soft factorization formulae involve colour correlations (see Eqs. (12) and (26)). As discussed at the end of Sect. 3, in the case of processes with two or three hard external partons, the correlations are completely given in terms of the products of the colour-charge 
factors $\boldsymbol{T}_{i} \cdot \boldsymbol{T}_{j}$. In this case, moreover, these colour-charge factors can be expressed in terms of the Casimir operators of the hard partons. Therefore the colour algebra can explicitly be carried out and we can obtain QED-like factorization formulae. We first recall the relations between colour-charge factors and Casimir operators and then give the factorization formulae.

When there are only two hard partons in the amplitude, we can use colour conservation $\left(\boldsymbol{T}_{1}=-\boldsymbol{T}_{2}\right)$ to obtain

$$
\boldsymbol{T}_{1} \cdot \boldsymbol{T}_{2}\left|\mathcal{M}\left(p_{1}, p_{2}\right)\right\rangle=-\boldsymbol{T}_{1}^{2}\left|\mathcal{M}\left(p_{1}, p_{2}\right)\right\rangle=-C_{1}\left|\mathcal{M}\left(p_{1}, p_{2}\right)\right\rangle=-C_{2}\left|\mathcal{M}\left(p_{1}, p_{2}\right)\right\rangle,
$$

where $C_{1}=C_{2}$ is the Casimir of the hard partons. When the hard partons are three, the colour algebra can still be performed in closed form. Let us consider, for instance, the correlation term $\boldsymbol{T}_{1} \cdot \boldsymbol{T}_{2}$. Using colour conservation $\left(\boldsymbol{T}_{3}=-\boldsymbol{T}_{1}-\boldsymbol{T}_{2}\right)$, its action on the ket $\left|\mathcal{M}\left(p_{1}, p_{2}, p_{3}\right)\right\rangle$ is

$$
2 \boldsymbol{T}_{1} \cdot \boldsymbol{T}_{2}\left|\mathcal{M}\left(p_{1}, p_{2}, p_{3}\right)\right\rangle=\left(\boldsymbol{T}_{3}^{2}-\boldsymbol{T}_{1}^{2}-\boldsymbol{T}_{2}^{2}\right)\left|\mathcal{M}\left(p_{1}, p_{2}, p_{3}\right)\right\rangle=\left(C_{3}-C_{1}-C_{2}\right)\left|\mathcal{M}\left(p_{1}, p_{2}, p_{3}\right)\right\rangle
$$

and thus $\boldsymbol{T}_{1} \cdot \boldsymbol{T}_{2}$ is again given in terms of the Casimir invariants $C_{i}$. The same can be done for the other products $\boldsymbol{T}_{1} \cdot \boldsymbol{T}_{3}$ and $\boldsymbol{T}_{2} \cdot \boldsymbol{T}_{3}$.

When the number of hard partons is four or more, colour correlations cannot be avoided. In fact the number of independent equations following from colour conservation is not sufficient to express the products $\boldsymbol{T}_{i} \cdot \boldsymbol{T}_{j}$ in terms of Casimir invariants.

The colour-algebra results in Eqs. (55) and (56) can be inserted in Eqs. (12), (24) and (26) to straightforwardly obtain the soft limit of the squared amplitudes with a soft gluon and two or three hard partons (plus any number of colourless partons). In both cases, the contributions from Eqs. (12) and (23) reconstruct the one-loop expansion of the all-order squared amplitude $|\mathcal{M}(\{p\})|^{2}$ in Eq. (酉), and we obtain the following factorization formulae

$$
\begin{gathered}
\left|\mathcal{M}\left(q, p_{1}, p_{2}\right)\right|^{2} \simeq g_{\mathrm{S}}^{2} \mu^{2 \epsilon} 4 C_{1} \mathcal{I}_{12}(q)\left|\mathcal{M}\left(p_{1}, p_{2}\right)\right|^{2} \\
\left|\mathcal{M}\left(q, p_{1}, p_{2}, p_{3}\right)\right|^{2} \simeq g_{\mathrm{S}}^{2} \mu^{2 \epsilon} 2\left[2 C_{1} \mathcal{I}_{12}(q)+C_{A}\left(\mathcal{I}_{23}(q)+\mathcal{I}_{13}(q)-\mathcal{I}_{12}(q)\right)\right]\left|\mathcal{M}\left(p_{1}, p_{2}, p_{3}\right)\right|^{2}
\end{gathered}
$$

where the kinematic factor $\mathcal{I}_{i j}(q)$ includes the loop corrections to the eikonal function $\mathcal{S}_{i j}(q)$ in Eq. (13). To one-loop accuracy, we find

$$
\mathcal{I}_{i j}(q)=\mathcal{S}_{i j}(q) \cdot\left\{1-C_{A} \frac{g_{\mathrm{S}}^{2}}{8 \pi^{2}} \frac{1}{\epsilon^{2}} \frac{\Gamma^{4}(1-\epsilon) \Gamma^{3}(1+\epsilon)}{\Gamma^{2}(1-2 \epsilon) \Gamma(1+2 \epsilon)}\left[4 \pi \mu^{2} \mathcal{S}_{i j}(q)\right]^{\epsilon}+\mathcal{O}\left(g_{\mathrm{S}}^{4}\right)\right\}
$$

where we have used Eq. (54). Note that in the amplitude with three hard partons, two of them (say, the ones with momenta $p_{1}$ and $p_{2}$ ) have to form a particle-antiparticle pair (either a $q \bar{q}$ pair or a gluon pair) and the third one (with momentum $p_{3}$ ) has to be a gluon. In Eq. (58) we have therefore set $C_{1}=C_{2}$ and $C_{3}=C_{A}$.

The results in Eqs. (57) and (58), when combined with the analogous QED-like factorization formulae for the emission of two soft gluons [6, 7] and of two [13, 14] and three [6, 
7] collinear partons, can be used to perform cross section calculations at NNLO for several processes. For instance, Eq. (57) is relevant to 2-jet production in $e^{+} e^{-}$annihilation and for the production of Drell-Yan, photon and vector-boson pairs in hadron collisions, while Eq. (58) can be used for the production of 3 jets in $e^{+} e^{-}$annihilation, $(2+1)$ jets in deep-inelastic lepton-hadron collisions and (vector boson + jet) in hadron collisions. Owing to the high precision of the data from $e^{+} e^{-}$-collider experiments, improved theoretical calculations for 3 -jet production are highly demanded. The fact that colour and kinematics factors are completely disentangled in the singular limits of the corresponding matrix elements certainly simplifies the structure of these calculations.

\section{Acknowledgements.}

We would like to thank Vittorio Del Duca and Zoltan Kunszt for comments.

\section{References}

1. S. Catani et al., hep-ph/0005025, in the Proceedings of the CERN Workshop on Standard Model Physics (and more) at the LHC, Eds. G. Altarelli and M.L. Mangano, CERN 2000-04, Geneva 2000.

2. S. Catani et al., hep-ph/0005114, to be published in the Proceedings of the Les Houches Workshop on Physics at TeV Colliders, Eds. P. Aurenche et al.

3. G. Altarelli and G. Parisi, Nucl. Phys. B126 (1977) 298.

4. A. Bassetto, M. Ciafaloni and G. Marchesini, Phys. Rep. 100 (1983) 201; Yu.L. Dokshitser, V.A. Khoze, A.H. Mueller and S.I. Troian, Basics of Perturbative QCD (Editions Frontières, Gif-sur-Yvette, 1991) and references therein.

5. R.K. Ellis, W.J. Stirling and B.R. Webber, $Q C D$ and collider physics (Cambridge University Press, Cambridge, 1996) and references therein.

6. J.M. Campbell and E.W.N. Glover, Nucl. Phys. B527 (1998) 264.

7. S. Catani and M. Grazzini, Nucl. Phys. B570 (2000) 287.

8. Z. Bern and G. Chalmers, Nucl. Phys. B447 (1995) 465.

9. D.A. Kosower, Nucl. Phys. B552 (1999) 319.

10. S. Catani and M. Grazzini, Phys. Lett. 446B (1999) 143.

11. V. Del Duca, A. Frizzo and F. Maltoni, Nucl. Phys. B568 (2000) 211.

12. Z. Bern, V. Del Duca and C.R. Schmidt, Phys. Lett. B445 (1998) 168.

13. D.A. Kosower and P. Uwer, Nucl. Phys. B563 (1999) 477.

14. Z. Bern, V. Del Duca, W.B. Kilgore and C.R. Schmidt, Phys. Rev. D60 (1999) 116001.

15. F.A. Berends and W.T. Giele, Nucl. Phys. B313 (1989) 595. 
16. S. Catani, Phys. Lett. 427B (1998) 161.

17. Z. Bern and D.A. Kosower, Nucl. Phys. B362 (1991) 389; Z. Kunszt, A. Signer and Z. Trócsányi, Phys. Lett. B336 (1994) 529; Z. Bern, L. Dixon and D. A. Kosower, Nucl. Phys. B437 (1995) 259.

18. G. 't Hooft and M. Veltman, Nucl. Phys. B44 (1972) 189.

19. G. Bollini and J.J. Giambiagi, Nuovo Cimento 12B (1972) 20; J.F. Ashmore, Nuovo Cimento Lett. 4 (1972) 289; G.M. Cicuta and E. Montaldi, Nuovo Cimento Lett. 4 (1972) 329.

20. W. Siegel, Phys. Lett. 84B (1979) 193 and 94B (1980) 37; D.M. Capper, D.R.T. Jones and P. van Nieuwenhuizen, Nucl. Phys. B167 (1980) 479; L.V. Avdeev and A.A. Vladimirov, Nucl. Phys. B219 (1983) 262.

21. Z. Bern and D.A. Kosower, Nucl. Phys. B379 (1992) 451.

22. Z. Kunszt, A. Signer and Z. Trócsányi, Nucl. Phys. B411 (1994) 397.

23. S. Catani, M.H. Seymour and Z. Trócsányi, Phys. Rev. D 55 (1997) 6819.

24. S. Catani and M.H. Seymour, Nucl. Phys. B485 (1997) 291 (E ibid. B510 (1998) $503)$.

25. D.R. Yennie, S.C. Frautschi and H. Suura, Ann. Phys. 13 (1961) 379; G. Grammer and D.R. Yennie, Phys. Rev. D8 (1973) 4332.

26. W.T. Giele and E.W.N. Glover, Phys. Rev. D 46 (1992) 1980.

27. Z. Kunszt and D.E. Soper, Phys. Rev. D 46 (1992) 192.

28. Z. Kunszt, A. Signer and Z. Trócsányi, Nucl. Phys. B420 (1994) 550.

29. S. Catani and M. Ciafaloni, Nucl. Phys. B249 (1985) 301.

30. S. Catani, M. Ciafaloni and G. Marchesini, Nucl. Phys. B264 (1986) 588.

31. S. Catani and M.H. Seymour, Phys. Lett. 378B (1996) 287.

32. A. Bassetto, G. Nardelli and R. Soldati, Yang-Mills theories in algebraic noncovariant gauges: Canonical quantization and renormalization, (World Scientific, Singapore, 1991); G. Leibbrandt, Noncovariant gauges: Quantization of Yang-Mills and ChernSimons theory in axial-type gauges (World Scientific, Singapore, 1994). 